\title{
IFNY and TNFa synergistically induce apoptosis of mesenchymal stem/stromal cells via the induction of nitric oxide
}

\author{
Xiaolei Li ${ }^{\dagger}$, Bingxue Shang ${ }^{\dagger}$, Ya-nan Li, Yufang Shi ${ }^{*}$ and Changshun Shao ${ }^{*}$
}

\begin{abstract}
Background: Mesenchymal stem/stromal cells (MSCs) have been widely used to treat various inflammatory diseases. The immunomodulatory capabilities of MSCs are usually licensed by inflammatory cytokines and may vary depending on the levels and the types of inflammatory cytokines. However, how the inflammatory microenvironment affects the fate of MSCs remains elusive. Here we characterized the molecular mechanism underlying the apoptosis of mouse MSCs triggered by the synergistic action of IFNY and TNFa.

Methods: We isolated and expanded MSCs by flushing the femoral and tibial bone marrow of wild-type, iNOS ${ }^{-1-}$, and $\mathrm{Fas}^{-/-}$mice. BM-MSCs were treated with IFNY and TNFa in vitro, and cell viability was evaluated by a CCK-8 kit. Apoptosis was assessed by Annexin V/propidium iodide-stained flow cytometry. Expression of genes related to apoptosis and endoplasmic reticulum (ER) stress was measured by reverse transcription-polymerase chain reaction (RT-PCR). Apoptosis and autophagy-related proteins were examined by Western blot analysis.
\end{abstract}

Results: IFNY and TNFa synergistically trigger apoptosis of mouse BM-MSCs. The two cytokines were shown to stimulate the expression of inducible nitric oxide synthase (iNOS) and consequently the generation of nitric oxide (NO), which is required for the apoptosis of mouse BM-MSCs. The two cytokines similarly induced apoptosis in $\mathrm{Fas}^{-1-}$ BM-MSCs. iNOS and NO were shown to upregulate Fas in mouse MSCs and sensitize them to Fas agonist-induced apoptosis. Moreover, NO stimulated by IFNY/TNFa impairs autophagy, which aggravates ER stress and promotes apoptosis.

Conclusions: IFNץ/TNFa-induced apoptosis in mouse MSCs is mediated by NO. Our findings shed new light on cytokine-induced apoptosis of MSCs and have implications in MSC-based therapy of inflammatory diseases.

Keywords: Mesenchymal stem/stromal cells, Inflammation, iNOS, Nitric oxide, Autophagy, Apoptosis

\section{Background}

Mesenchymal stem/stromal cells (MSCs) are multipotent stem cells that exist in almost all types of tissues, including bone marrow, umbilical cord, muscle, fat, and dermis [1]. Depending on the stimuli and the culture conditions employed, MSCs are capable of differentiating into osteoblasts, adipocytes, and chondrocytes [2]. In addition to their potential to differentiate into multiple cell lineages, MSCs also acquire potent immunoregulatory function when properly stimulated, which makes them suitable and highly effective for clinical

\footnotetext{
* Correspondence: yfshi@suda.edu.cn; shaoc@suda.edu.cn

${ }^{+}$Xiaolei Li and Bingxue Shang contributed equally to this work.

The First Affiliated Hospital of Soochow University and State Key Laboratory of Radiation Medicine and Protection, Institutes for Translational Medicine, Soochow University, Suzhou 215123, Jiangsu, China
}

applications in treating a variety of human disease, such as graft-versus-host disease (GvHD) and systemic lupus erythematosus (SLE) [1-3].

The inflammatory microenvironment plays a crucial role in the acquisition of the immunoregulatory capability by MSCs [4]. Mechanistic studies exploring the immunosuppressive effects of MSCs on $\mathrm{T}$ cells have revealed that a combination of interferon (IFN)- $\gamma$ and any of three other pro-inflammatory cytokines, tumor necrosis factor (TNF)- $\alpha$, interleukin (IL)- $1 \alpha$, and IL-1 $\beta$, can activate MSCs to exert immunosuppressive effects in mice [5]. These cytokine combinations provoke the expression of high levels of several chemokines and inducible nitric oxide synthase (iNOS) by MSCs [5]. The T cells are recruited to the proximity of the MSCs by 
chemokines and are killed by nitric oxide (NO). Unlike murine MSCs which utilize iNOS, human MSCs employ indoleamine 2,3-dioxygenase (IDO), the tryptophancatabolic enzyme, to suppress $\mathrm{T}$ cells [1].

While exogenous MSCs have been widely used to repair tissue damage and to control inflammation via the production of chemokines and NO or IDO, the infused MSCs tend to have a very limited lifespan [6]. How those MSCs disappear in vivo remains largely elusive. One study showed that when exposed to IFN $\gamma / \mathrm{TNF} \alpha$ mouse MSCs could undergo apoptosis mediated by Fas (CD95/ Apo-1) and were thus compromised in their bone-repairing function [7]. IFNY plus TNF $\alpha$ were also shown to induce autophagy in MSCs and inhibition of autophagy could augment the immunosuppressive effect on T cells by MSCs $[8,9]$. However, survival of MSCs was recently shown to be dispensable for the immunosuppressive effect [10]. Instead, MSCs are actively induced to undergo perforin-dependent apoptosis by recipient cytotoxic cells and the apoptosis of MSCs is essential for their immunosuppression in a murine model of GvHD. These studies suggest that fate of MSCs may modulate the therapeutic effects of MSCs in a context-dependent manner. Since iNOS is drastically induced by IFN $\gamma$ plus TNF $\alpha$ in MSCs and NO is responsible for the cell cycle arrest and apoptosis of T cells, we hypothesized that $\mathrm{NO}$ might also play a role in the death of murine MSCs when exposed to IFN $\gamma$ and TNF $\alpha$. The finding that $\mathrm{NO}$ can impair pro-survival autophagy also renders support to this notion [11]. We therefore investigated apoptosis induced by IFNY plus TNF $\alpha$ in cultured mouse bone-marrow (BM)-MSCs (BM-MSCs). We found that $\mathrm{NO}$ indeed mediates the death of murine MSCs upon exposure to IFN $\gamma$ and TNF $\alpha$.

\section{Methods \\ MSC isolation and culture}

MSCs were generated using our previously described protocol [5]. Briefly, tibia and femur bone marrow of 6-week-old wild-type, $\mathrm{iNOS}^{-/-}$, and $\mathrm{Fas}^{-/-}$mice was harvested. Cells were cultured in DMEM supplemented with $10 \%$ FBS, $2 \mathrm{mM}$ glutamine, $100 \mathrm{U} / \mathrm{ml}$ penicillin, and $100 \mu \mathrm{g} / \mathrm{ml}$ streptomycin (complete medium, all from Invitrogen, Carlsbad, CA, USA). All non-adherent cells were removed after $24 \mathrm{~h}$, and adherent cells were maintained. Medium was changed every 3 days. To obtain MSC clones, cells at confluence were harvested and seeded into 96-well plates by limited dilution. Individual clones were then picked and expanded. These MSCs were capable of differentiating into adipocytes and osteocytes under the respective differentiation conditions. Cells were used before the 20th passage.

\section{Cell viability assay}

Cell viability was determined using a CCK-8 Kit (Dojindo, Japan). Cells were seeded into 96-well plates at $2 \times 10^{3}$ cells/well and cultured for $24 \mathrm{~h}$ at $37^{\circ} \mathrm{C}$ with $5 \% \mathrm{CO}_{2}$. After treatment with PBS as a control or IFN $\gamma$ and TNF $\alpha$ at varying concentrations for the indicated times, $10 \mu \mathrm{l}$ of CCK- 8 solutions was added to each well of the plate. The cells were incubated for one more hour, and the absorbance of the samples $(450 \mathrm{~nm})$ was determined by using a scanning multi-well spectrophotometer. The cell viability was calculated using the following formula: relative cell viability $(\%)=\left(\right.$ absorbance $_{450 \mathrm{~nm}}$ of treated group - absorbance $_{450 \mathrm{~nm}}$ of blank)/(absorbance $450 \mathrm{~nm}$ of control group absorbance $_{450} \mathrm{~nm}$ of blank). The results from three independent experiments in triplicates are presented.

\section{Real-time PCR}

Total RNA from each sample was extracted by the Trizol reagent (Thermo Fisher). Purity was addressed by the absorbance ratio at 260 and $280 \mathrm{~nm}$. First-strand cDNA synthesis was performed using PrimeScript ${ }^{\text {Tw }}$ RT Master Mix according to the manufacturer's instructions (TaKaRa Biotech, Dalian, China). The levels of mRNA of genes of interest were measured by real-time PCR (7900 HT by Applied Biosystems, Foster City, CA, USA) using SYBR Green Master Mix (TaKaRa Biotech, Dalian, China). Total amount of mRNA was normalized to endogenous $\beta$-actin mRNA. The primers of the target genes were designed as shown in Table 1.

\section{Western blotting}

Cells were washed twice with ice-cold PBS, harvested and lysed in the RIPA buffer (Millipore, Temecular, CA, USA) containing a cocktail of protease inhibitors (Roche, Nutley, NJ, USA) and PMSF for 30 min on ice. Lysates were clarified by centrifugation at $16000 \times g$ for $15 \mathrm{~min}$ and heated in sodium dodecyl sulfate sample buffer at $95^{\circ} \mathrm{C}$ for $10 \mathrm{~min}$. Protein concentration of the supernatant was determined by the Bradford assay (Bio-Rad, Hercules, CA, USA). Protein samples were separated on a polyacrylamide gel, and separated proteins were electroblotted onto polyvinylidene difluoride membranes. Specific proteins were revealed by mouse and rabbit antibodies by overnight incubation at $4{ }^{\circ} \mathrm{C}$, followed by chemiluminescent detection according to the manufacturer's instructions.

\section{Annexin-V/propidium iodide flow cytometric analysis}

The apoptosis induced by cytokines and/or $S$-nitroso-$\mathrm{N}$-acetyl-penicillamine (SNAP) was assessed by staining cells with an Annexin V-fluorescein isothiocyanate (APC) Apoptosis Detection Kit (BD Biosciences). The cells were collected and then washed with cold PBS twice. Then, they were resuspended in $100 \mu \mathrm{l}$ of Annexin 
Table 1 Gene-specific primers for qRT-PCR

\begin{tabular}{|c|c|}
\hline Genes & Oligonucleotide sequence $\left(5^{\prime}-3^{\prime}\right)$ \\
\hline \multirow[t]{2}{*}{$\beta$-actin } & F: CAACGAGCGGTTCCGATG \\
\hline & R: GCCACAGGATTCCATACCCA \\
\hline \multirow[t]{2}{*}{ Atf4 } & F: CGGGTGTCCCTITCCTCTTC \\
\hline & R: TGAAGAGCGCCATGGCTTAG \\
\hline \multirow[t]{2}{*}{ Bip/GRP78 } & F: GTGTGTGAGACCAGAACCGT \\
\hline & R: ACAGTGAACTTCATCATGCCG \\
\hline \multirow[t]{2}{*}{ Erdj4/Dnajb9 } & F: GGGGCGCACAGGTTATTAGAA \\
\hline & R: TCTGAGGCAGACTTTGGCAC \\
\hline \multirow[t]{2}{*}{ Chop } & F: GGAACCTGAGGAGAGAGTGTT \\
\hline & R: AAGGTGAAAGGCAGGGACTC \\
\hline \multirow[t]{2}{*}{ Xbp1 } & F: GCAGCAAGTGGTGGATTTGG \\
\hline & R: CCTTACTCCACTCCCCTTGG \\
\hline \multirow[t]{2}{*}{ Fas } & F: GCTTGCTGGCTCACAGTTAAG \\
\hline & R: AGGTTGGTGTACCCCCATTC \\
\hline \multirow[t]{2}{*}{$\mathrm{BCl} 2$} & F: CACCCCTGGTGGACAACATC \\
\hline & R: ATAGTTCCACAAAGGCATCCCAG \\
\hline \multirow[t]{2}{*}{$\mathrm{BCl} 2 \mathrm{1} 11$} & F: GCCAGGCCTTCAACCACTAT \\
\hline & R: TGCAAACACCCTCCTTGTGT \\
\hline \multirow[t]{2}{*}{ Bax } & F: TGCTAGCAAACTGGTGCTCA \\
\hline & R: AGTAGGAGAGGAGGCCCAGC \\
\hline
\end{tabular}

$F$ forward primer, $R$ reverse primer

$\mathrm{V}$ binding buffer and incubated with $5 \mu \mathrm{l}$ of APC-conjugated Annexin V and $5 \mu \mathrm{l}$ of propidium iodide for $15 \mathrm{~min}$ in the dark. Annexin V binding buffer $(200 \mu \mathrm{l})$ was then added to each tube. Finally, the cells were examined using a BD FACS-Canto II flow cytometer (BD Biosciences, CA). All experiments were performed in triplicate and repeated three times independently.

\section{Statistical analysis}

The data are presented as the means \pm SD of three independent experiments. For the determination of statistical significance, a one-way analysis of variance (ANOVA) and a two-way ANOVA were performed using Prism software (version 7.0, GraphPad Software, La Jolla, CA). $P$ values $<0.05$ were considered statistically significant.

\section{Results}

IFN $Y$ and TNFa synergistically trigger apoptosis in mouse BM-MSCs

To investigate whether inflammatory cytokines induce apoptosis of MSCs, we cultured BM-MSCs in vitro with TNF $\alpha$, IFN $\gamma$, or the two in combination. Treatment of mouse BM-MSCs with TNF $\alpha$ or with IFN $\gamma$ alone did not substantially affect their viability (Fig. 1a). However, the two in combination significantly reduced MSC viability and induced massive cell death (Fig. 1a, b), which could be inhibited by the caspase inhibitor Z-VAD-FMK
(Fig. 1c). Among the important regulators of apoptosis are the pro- and anti-apoptotic proteins of the Bcl-2 family and the pro-apoptotic multidomain proteins Bax and Bak are needed for apoptosis mediated by mitochondria [12]. Oligomerization of Bax and Bak leads to formation of membrane pores through which apoptotic mediators, such as cytochrome $c$, are released to the cytoplasm and/or apoptosis-inducing factor (AIF) to the nucleus [12]. Once in the cytoplasm, cytochrome $c$ interacts with apoptotic protease activating factor to form the apoptosome, leading to pro-caspase cleavage and activation and subsequent cell death. We examined the effect of IFN $\gamma / \mathrm{TNF} \alpha$ treatment on the expression of representative pro- and anti-apoptotic genes in MSCs by quantitative RT-PCR analysis. As shown in Fig. 1d, treatment of mouse BM-MSCs with IFN $\gamma / \mathrm{TNF} \alpha$ led to higher expression of pro-apoptotic genes and lower expression of anti-apoptotic genes in BM-MSCs compared with controls. These data indicate that IFN $\gamma / \mathrm{TNF} \alpha$ synergistically induce BM-MSC apoptosis.

\section{BM-MSC apoptosis induced by IFNY and TNFa is mediated by $\mathrm{NO}$}

We examined the effects of TNF $\alpha$, IFN $\gamma$, or the two in combination on the expression of NOS in MSCs and revealed that the level of iNOS was synergistically enhanced by IFN $\gamma / \mathrm{TNF} \alpha$, which corresponded to their ability to induce cell death (Fig. 2a, b). Endogenous NO is synthesized from L-arginine by a family of NOS in a two-step oxidation process. An excess of NO resulting in nitrosative stress is believed to play a causal role in demise of several type of cells, including neuronal cells [13], T cells [14], dendritic cells [15], and microglial cells [16]. We therefore tested whether NO is a crucial mediator of IFN $\gamma / \mathrm{TNF} \alpha$-induced BM-MSC apoptosis. Notably, blocking iNOS activity by the NOS inhibitor $\mathrm{N}^{\mathrm{G}}$-monomethyl-L-arginine (L-NMMA) could protect BM-MSCs from IFN $\gamma / \mathrm{TNF} \alpha$-induced cell death (Fig. 2c). The increased apoptosis induced by IFN $\gamma / \mathrm{TNF} \alpha$ was also prevented in BM-MSCs derived from iNOS ${ }^{-/-}$mice (Fig. 2b-d). Collectively, these results demonstrated that $\mathrm{NO}$ plays a critical role in IFN $\gamma / \mathrm{TNF} \alpha$-induced apoptosis in mouse BM-MSCs.

To further substantiate this notion, we examined whether direct application of $\mathrm{NO}$ also induces apoptosis in MSCs via mitochondrial pathway. BM-MSCs were treated with the NO donor $S$-nitroso- $N$-acetyl-penicillamine (SNAP). As shown in Fig. 2e, this treatment caused cell death in BM-MSCs not treated with IFN $\gamma / T N F \alpha$. SNAP had similar effect on iNOS $^{-/-}$BM-MSCs independent of IFN $\gamma /$ TNF $\alpha$ (Fig. 2f). Death was also accompanied by Bax and Bak activation and activation of caspase-3 (data not shown). Notably, assessment of apoptotic-related gene expression in BM-MSCs revealed 

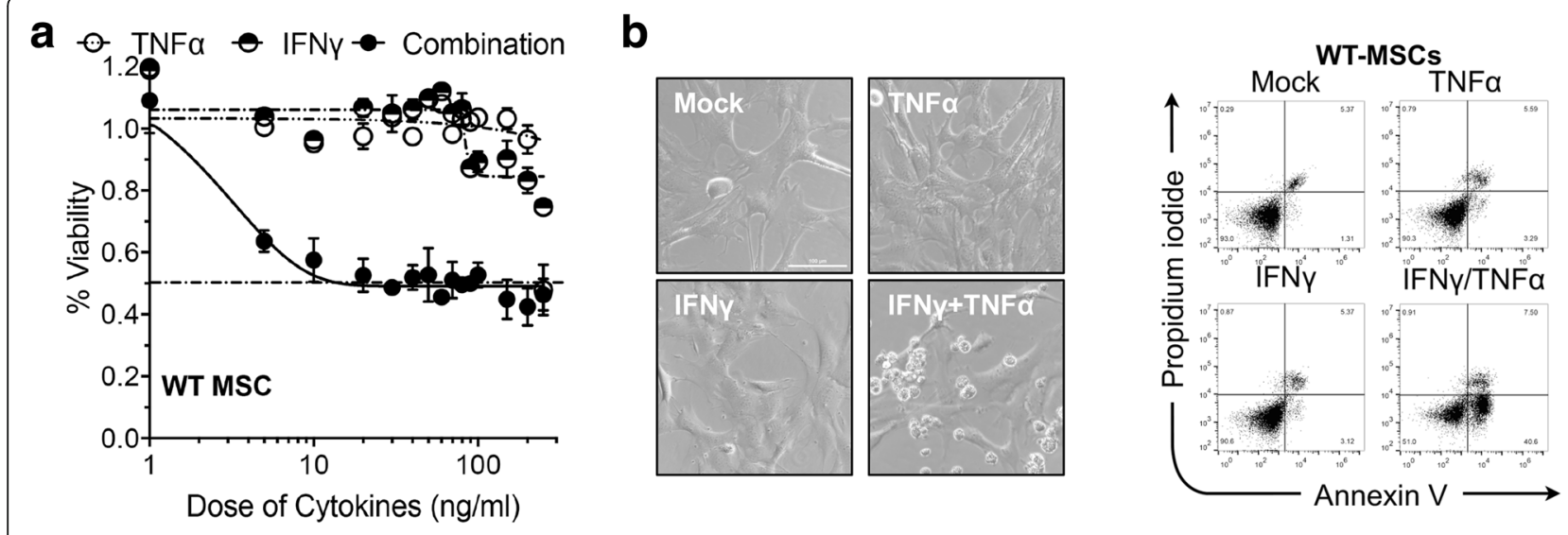

C

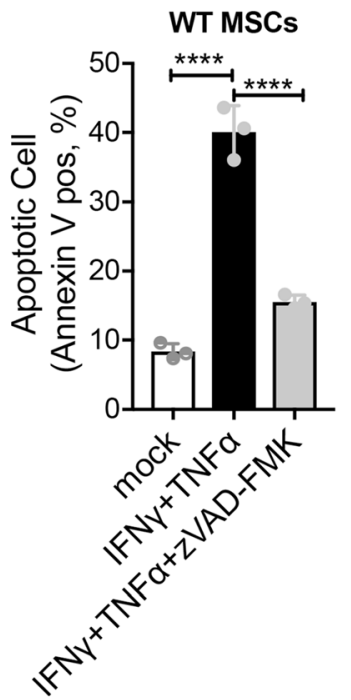

d

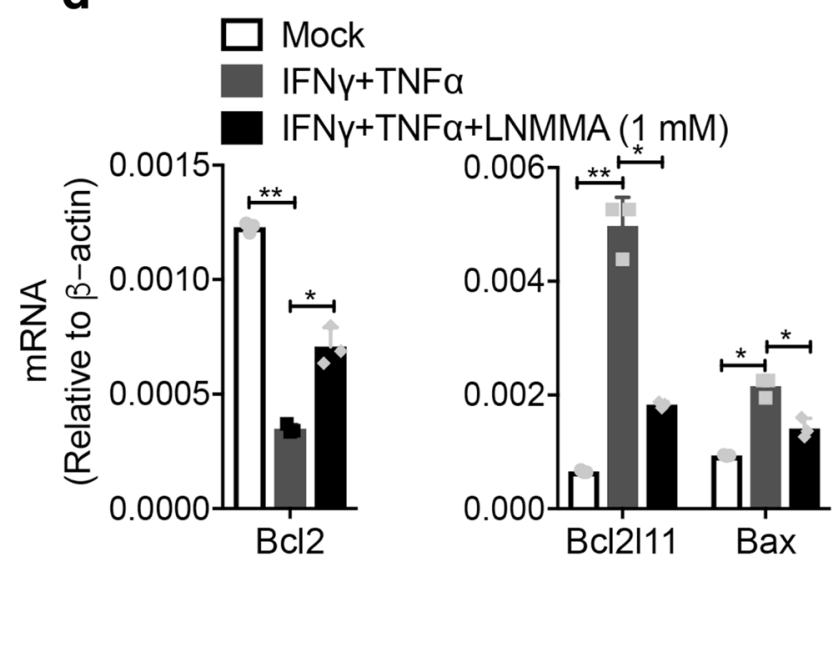

\section{WT MSCs}

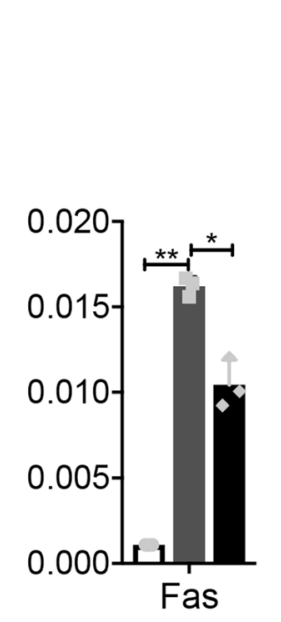

Fig. 1 IFNY and TNFa synergistically induced apoptosis in mouse BM-MSCs. a Percentage of wild-type BM-MSC cell survival after treatment with various concentrations of TNFa or IFNy alone or a combination of these two cytokines. $\mathbf{b}$ Wild-type BM-MSCs were treated with IFNy/TNFa (10 ng/ml each) for $48 \mathrm{~h}$. Apoptotic cells were analyzed using flow cytometry. The percentage of Annexin V-positive cells relative to untreated controls is indicated in a bar chart. c Wild-type BM-MSCs were treated with IFNy/TNFa ( $10 \mathrm{ng} / \mathrm{ml}$ each) in the absence or presence (1 h preincubation) of zVAD-FMK for $48 \mathrm{~h}$. Apoptotic cells were analyzed using flow cytometry. The percentage of Annexin V-positive cells relative to untreated controls is indicated in a bar chart. d BM-MSCs were treated with IFNY/TNFa (10 ng/ml each) in the absence or presence (1 h pre-incubation) of L-NMMA (1 mM) for $24 \mathrm{~h}$. Pro-apoptotic and anti-apoptotic transcripts were quantified by real-time $P C R .{ }^{*} P<0.05,{ }^{* *} P<0.01,{ }^{* * * *} P<0.0001$

that SNAP led to higher expression of pro-apoptotic genes and lower expression of anti-apoptotic genes in BM-MSCs compared with controls (Fig. 2g). These results indicate that $\mathrm{NO}$ mediates the cytotoxic effect of IFN $\gamma / \mathrm{TNF} \alpha$ on BM-MSCs.

\section{Fas is dispensable for the IFN $/$ TNFa-induced BM-MSC apoptosis}

Fas is a classical type I transmembrane receptor and belongs to the TNF receptor superfamily [17]. It was reported that Fas internalization and clustering mediate the apoptosis in BM-MSC by activation of caspase- 8 or caspase-3 in the presence of IFN $\gamma / \mathrm{TNF} \alpha$ [7]. We further validated whether Fas signaling is required for IFN $\gamma / \mathrm{TNF} \alpha$-induced BM-MSC apoptosis using $\mathrm{Fas}^{-/-}$
BM-MSCs. Unexpectedly, these two pro-inflammatory cytokines in combination could markedly reduce cell viability (Fig. 3a) and induce massive cell death in $\mathrm{Fas}^{-1-}$ BM-MSCs (Fig. 3b). These data suggest that Fas receptor may not be required for IFN $\gamma / \mathrm{TNF} \alpha$-induced BMMSC apoptosis. Quantitative RT-PCR analysis also revealed increased expression of pro-apoptotic genes and reduced expression of anti-apoptotic genes in $\mathrm{Fas}^{-1-}$ BM-MSCs (Fig. 3c).

To further investigate whether apoptosis in $\mathrm{Fas}^{-/-}$ BM-MSCs triggered by the combination of IFN $\gamma / \mathrm{TNF} \alpha$ depends on NO production, we examined the effect of IFN $\gamma / \mathrm{TNF} \alpha$ on the expression of NOS in $\mathrm{Fas}^{-/-}$ BM-MSCs and observed that the level of iNOS was also stimulated. Notably, apoptosis in $\mathrm{Fas}^{-/-}$BM-MSCs 


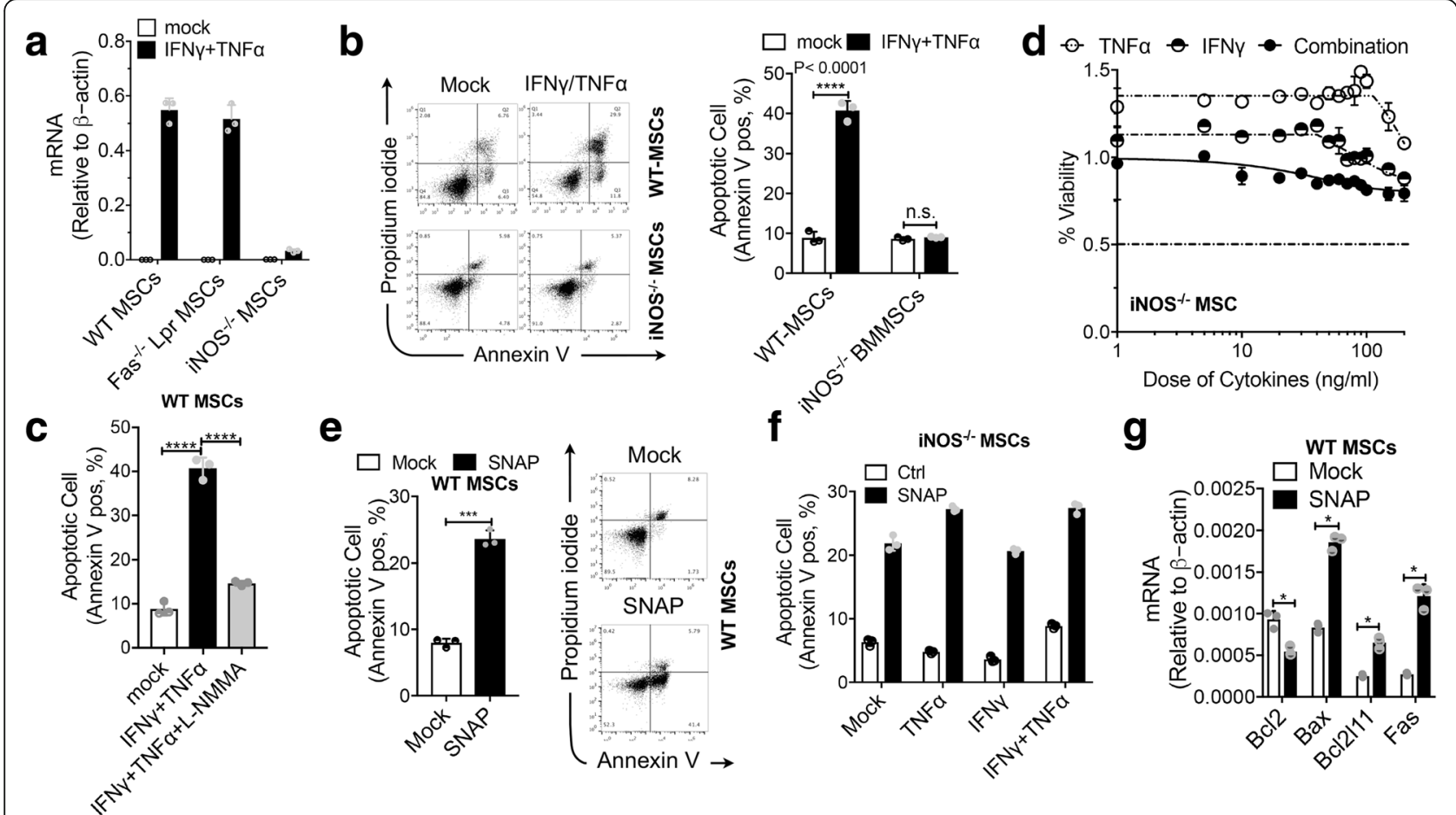

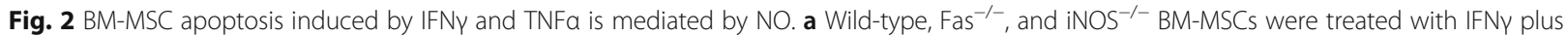

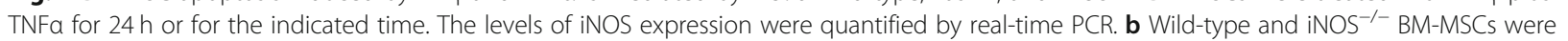
treated with IFNY/TNFa (10 ng/ml each) for $48 \mathrm{~h}$. Apoptotic cells were analyzed using flow cytometry. The percentage of Annexin V-positive cells relative to untreated controls is indicated in a bar chart. c Wild-type BM-MSCs were treated with IFNY/TNFa (10 ng/ml each) in the absence or presence ( $1 \mathrm{~h}$ pre-incubation) of L-NMMA (1 mM) for $48 \mathrm{~h}$. Apoptotic cells were analyzed using flow cytometry. The percentage of Annexin V-positive cells relative to untreated controls is indicated in a bar chart. $\mathbf{d}$ Percentage of iNOS ${ }^{-1}$ BM-MSC survival after treatment with various concentrations of different cytokines. e Wild-type MSCs were treated with SNAP $(0.5 \mathrm{mM})$ for $48 \mathrm{~h}$. Apoptotic cells were analyzed using flow cytometry. The percentage of Annexin V-positive cells relative to untreated controls is indicated in a bar chart. $\mathbf{f}$ iNOS ${ }^{-1-}$ BM-MSCs were treated with the indicated conditions for $48 \mathrm{~h}$. Apoptotic cells were analyzed using flow cytometry. The percentage of Annexin V-positive cells relative to untreated controls is indicated in a bar chart. $\mathbf{g}$ Wild-type BM-MSCs were treated with SNAP; pro-apoptotic and anti-apoptotic transcripts were quantified by real-time PCR. ${ }^{*} P<0.05,{ }^{* *} P<0.01,{ }^{* * *} P<0.001$, ${ }^{* * *} P<0.0001$

triggered by the combination of IFN $\gamma / \mathrm{TNF} \alpha$ could also be reversed by pretreatment with the NOS inhibitor L-NMMA (Fig. 3d). Additionally, assessment of the effect of L-NMMA on the expression of apoptosis-related genes in $\mathrm{Fas}^{-/-}$BM-MSCs also revealed that NOS inhibitor not only prevented the IFN $\gamma / \mathrm{TNF} \alpha$-induced increase in expression of pro-apoptotic genes, but also enhanced the level of anti-apoptotic genes in $\mathrm{Fas}^{-1-}$ BM-MSCs (Fig. 3e). Moreover, treatment of $\mathrm{Fas}^{-/-} \mathrm{BM}-\mathrm{MSCs}$ with the NO donor SNAP caused massive cell death (Fig. 3f). Assessment of the effects of SNAP on apoptotic-related gene expression in $\mathrm{Fas}^{-/-} \mathrm{BM}-\mathrm{MSC}$ revealed that SNAP led to higher expression of pro-apoptotic genes and lower expression of anti-apoptotic genes in BM-MSCs compared with controls (Fig. 3g). These data further suggest that Fas signaling might be not required for the IFN $\gamma / \mathrm{TNF} \alpha$-induced BM-MSC apoptosis and $\mathrm{NO}$, instead, plays a critical role in apoptosis of BM-MSCs triggered by IFN $\gamma / \mathrm{TNF} \alpha$.

\section{NO enhances Fas-mediated apoptosis in BM-MSCs}

Fas has been recognized as a death receptor, particularly in mediating non-specific $\mathrm{T}$ cell cytotoxicity and activation-induced cell death (AICD) in the peripheral immune system [18]. However, the induction of apoptosis by IFNy/TNFa in $\mathrm{Fas}^{-/-}$, but not in $\mathrm{iNOS}^{-1-}$, BM-MSCs argues against Fas playing a critical role in the apoptosis of BM-MSCs. Nevertheless, NO is known to serve as an intracellular second messenger to modify gene expression, and protein modification by $S$-nitrosylation is a common mechanism of NO-mediated signal transduction [19]. The role of NO on Fas receptor expression has been reported. The mechanism involved is NO inhibits Yin Yang 1 (YY1) DNA-binding activity through $S$-nitrosation and consequently results in upregulation of Fas expression [20, 21]. Thus, we next examined whether NO contributes to the IFN $\gamma / \mathrm{TNF} \alpha-$ induced sensitization of mouse BM-MSCs to Fas-mediated apoptosis. As shown in Figs. $1 \mathrm{~d}$ and $2 \mathrm{~g}$, IFN $\gamma / \mathrm{TNF} \alpha$-mediated upregulation of Fas expression in 


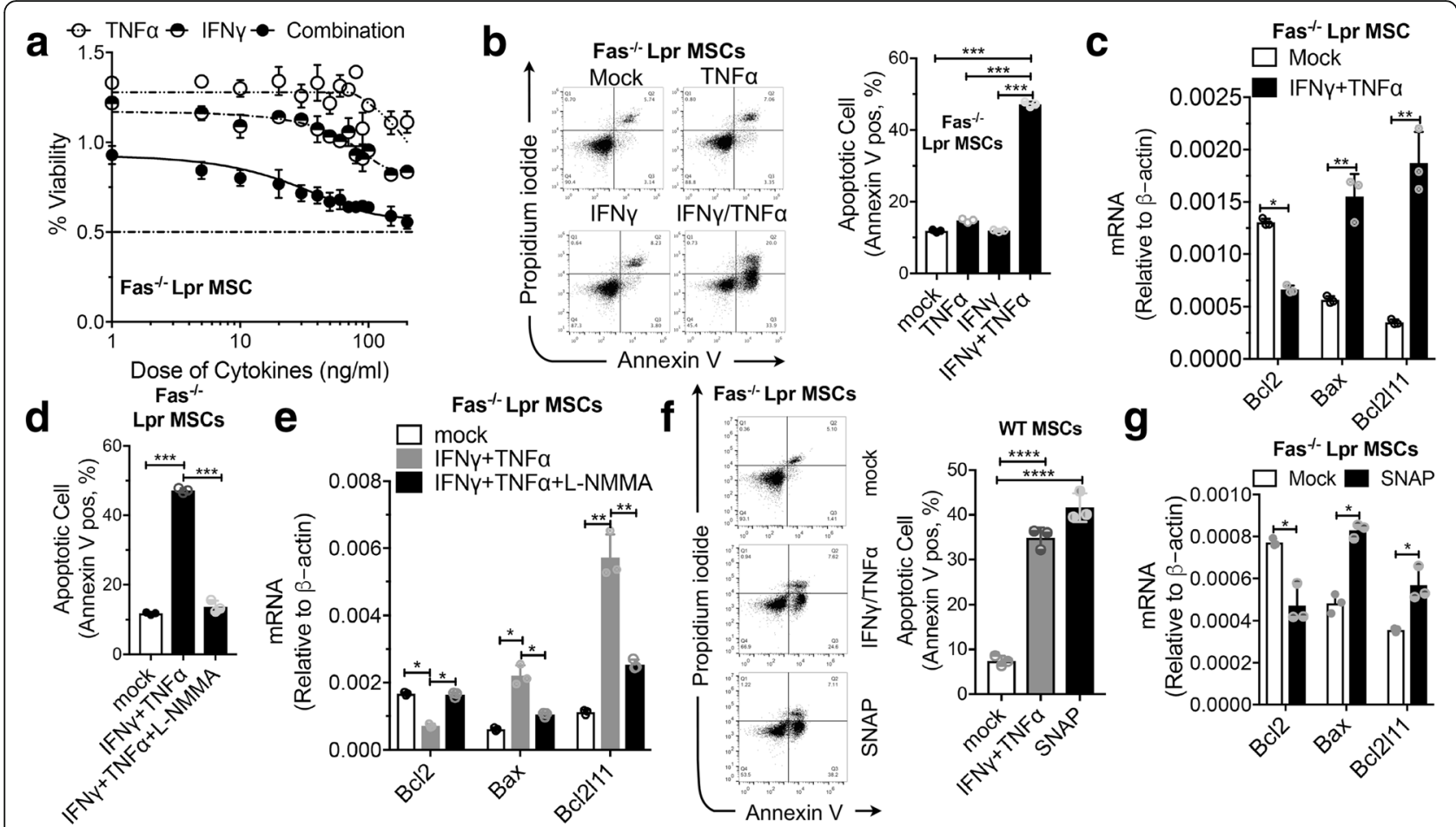

Fig. 3 Fas is not required for IFNY/TNFa-induced apoptosis in MSCs. a Percentage of Fas ${ }^{-1-}$ BM-MSC cell survival after treatment with various concentrations of different cytokines. $\mathbf{b} \mathrm{Fas}^{-1-}$ BM-MSCs were treated with the indicated cytokines for $48 \mathrm{~h}$, apoptotic cells were analyzed using flow cytometry. The percentage of Annexin V-positive cells relative to untreated controls is indicated in a bar chart. $\mathbf{c}$ Fas ${ }^{-1-}$ BM-MSCs were treated with the indicated cytokines for $24 \mathrm{~h}$; pro-apoptotic and anti-apoptotic transcripts were quantified by real-time PCR. $\mathbf{d}$ Fas ${ }^{-1-}$ BM-MSCs were treated with IFNY/TNFa $(10 \mathrm{ng} / \mathrm{ml}$ each) in the absence or presence (1 h pre-incubation) of L-NMMA (1 mM) for $48 \mathrm{~h}$. Apoptotic cells were analyzed using flow cytometry. The percentage of Annexin V-positive cells relative to untreated controls is indicated in a bar chart. e Fas ${ }^{-1-}$ BM-MSCs were treated as described in $\mathbf{d}$. Pro-apoptotic and anti-apoptotic transcripts were quantified by real-time PCR. $\mathbf{f}$ Fas ${ }^{-1-}$ BM-MSCs were treated

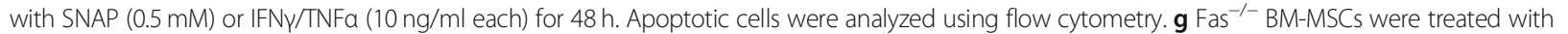
SNAP $(0.5 \mathrm{mM})$ for $24 \mathrm{~h}$; pro-apoptotic and anti-apoptotic transcripts were quantified by real-time $P C R$. ${ }^{*} P<0.05$, ${ }^{*} P<0.01$, ${ }^{* * *} P<0.001$

BM-MSCs was blockaded by NOS inhibitor, but restored by the NO donor (SNAP, $200 \mu \mathrm{M}$ ). Notably, the level of Fas induced by IFN $\gamma / \mathrm{TNF} \alpha$ was significantly reduced in iNOS $^{-1-}$ MSCs, compared with wild-type MSCs (Fig. 4a). These results indicate Fas induction by IFN $\gamma / \mathrm{TNF} \alpha$ in MSCs is possibly dependent on NO. We then examined the sensitivity of wild-type and $\mathrm{iNOS}^{-/-}$ BM-MSCs to Fas-mediated apoptosis using Fas agonistic antibody Jo2. As shown in Fig. 4b, apoptosis induced by a combination of IFN $\gamma / T N F \alpha$ was further enhanced by Jo2. As expected, sensitization to Fas-mediated apoptosis by IFN $\gamma /$ TNF $\alpha$ was significantly decreased in $\mathrm{iNOS}^{-/-}$MSCs. The role of the $\mathrm{NO}$ in the IFN $\gamma / \mathrm{TNF} \alpha$-mediated sensitization to Fas-induced apoptosis was corroborated by the use of SNAP, an exogenous source of NO that mimics the production of NO by iNOS (Fig. 4c). Our results herein with BM-MSCs clearly demonstrated that NO induced by IFN $\gamma / \mathrm{TNF} \alpha$, instead of protecting MSCs from Fas-induced apoptosis, synergized with Jo2 to induce apoptosis.

\section{Blockade of autophagy by iNOS sensitizes BM-MSCs to apoptosis}

Autophagy is a catabolic process aimed at restoring energy homeostasis through self-digestion of intracellular proteins and organelles to survive under starvation. It also functions to resist other types of stress that cause damage in organelles, such as the endoplasmic reticulum (ER) or mitochondria [22]. Notably, NO has been reported to impair autophagy by inhibiting the activity of $S$-nitrosylation substrates, JNK1 and IKK $\beta$ [11]. We thus evaluated the impact of autophagy inhibition on the survival of MSCs with or without exposure to TNF $\alpha / \mathrm{IFN} \gamma$. Inhibition of autophagy through 16-h exposure to chloroquine (CQ: $20 \mu \mathrm{M})$ decreased MSC cell viability and enhanced MSC apoptosis (Fig. 5a). Stimulating autophagy using the mTORC1 inhibitor rapamycin (Rap: $100 \mathrm{nM}$ ), on the other hand, could protect BM-MSCs against IFN $\gamma / \mathrm{TNF} \alpha$-induced BM-MSC apoptosis (Fig. 5a). The processing of LC3-I into LC3-II marks autophagosome formation. LC3-II accumulation can be detected in two situations: (i) autophagic activity is 

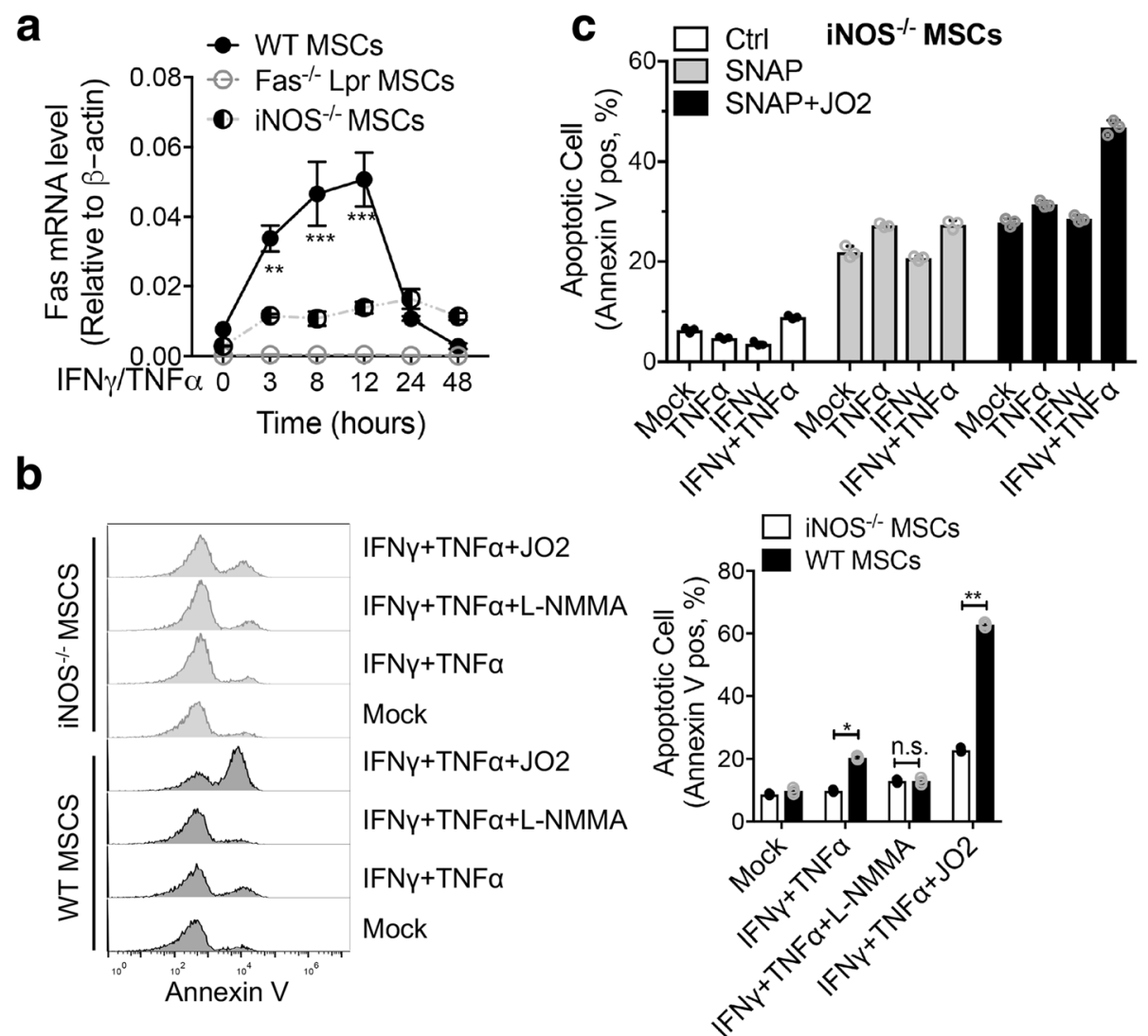

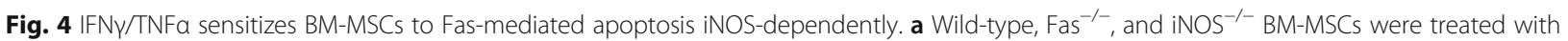
IFNY/TNFa for $24 \mathrm{~h}$ or for the indicated time. The levels of Fas expression were quantified by real-time PCR. $\mathbf{b}, \mathbf{c}$ Wild-type, and iNOS ${ }^{-1-}$, BM-MSCS were treated with the indicated conditions for $48 \mathrm{~h}$. Apoptotic cells were analyzed using flow cytometry. The percentage of Annexin V-positive cells relative to untreated controls is indicated in a bar chart. ${ }^{*} P<0.05,{ }^{* *} P<0.01$, ${ }^{* *} P<0.001$

stimulated and (ii) autophagic flux is blocked and vesicles accumulate [23]. Time-course of autophagy blockade using CQ allows monitoring of the autophagy flux. As expected, in control condition, autophagic flux blockade by CQ led to the accumulation of LC3-II and p62 due to the buildup of newly formed autophagosomes (Additional file 1: Figure S1). Further experiments were performed using rapamycin to stimulate autophagy. In absence of IFN $\gamma / \mathrm{TNF} \alpha$ cytokines, rapamycin elevated LC3-II levels in $\mathrm{iNOS}^{-1-}$, but not in wild-type, BM-MSCs (Fig. 5b). The $12 \mathrm{~h}$ pre-treatment with IFN $\gamma / \mathrm{TNF} \alpha$ cytokines abolished the effect of rapamycin on LC3-II in wild-type, but not in $\mathrm{iNOS}^{-1-}$, BM-MSCs (Fig. 5b), suggesting that the impairment of autophagic flux by cytokine exposure is dependent on the activity of iNOS. Augmentation of autophagy by rapamycin protected BM-MSCs against IFN $\gamma / \mathrm{TNF} \alpha$-induced BM-MSC apoptosis, as reflected by substantially attenuated activation of caspase-3 (Fig. 5b). These results suggest that NO may promote apoptosis of MSCs exposed to IFN $\gamma /$ TNF $\alpha$ by impairing the pro-survival autophagy.
ER stress is also considered a major cause of apoptosis in many biological processes. We speculated that cytokines might induce ER overload and ER stress, resulting in the unfolded protein response (UPR)-triggered apoptosis of MSCs. As expected, treatment of MSCs with a combination of IFN $\gamma$ and TNF $\alpha$ resulted in the upregulation of the levels of Atf4, Bip/GRP78, Chop, ERdj4/Dnajb9, and Xbp1, which are considered general ER stress biomarkers (Additional file 2: Figure S2a). Notably, ER stress induced by cytokines could be alleviated by NOS inhibitor, L-NMMA, as conveyed by downregulation of several general ER stress biomarkers (Additional file 2: Figure S2a). Similar results were also observed in $\mathrm{Fas}^{-/-} \mathrm{BM}-\mathrm{MSCs}$ (Additional file 2: Figure S2b). Autophagy may alleviate ER stress triggered by damaged organelles. To assess the relationship between ER stress and autophagy, we studied the expression of the known ER stress marker. IFNY/ TNF $\alpha$-induced ER stress marker expressions were increased in presence of $C Q$, suggesting that blocking autophagy induced ER stress (Additional file 3: Figure S3). Interestingly, rapamycin exposure decreased IFN $\gamma / \mathrm{TNF} \alpha$-induced ER stress gene 


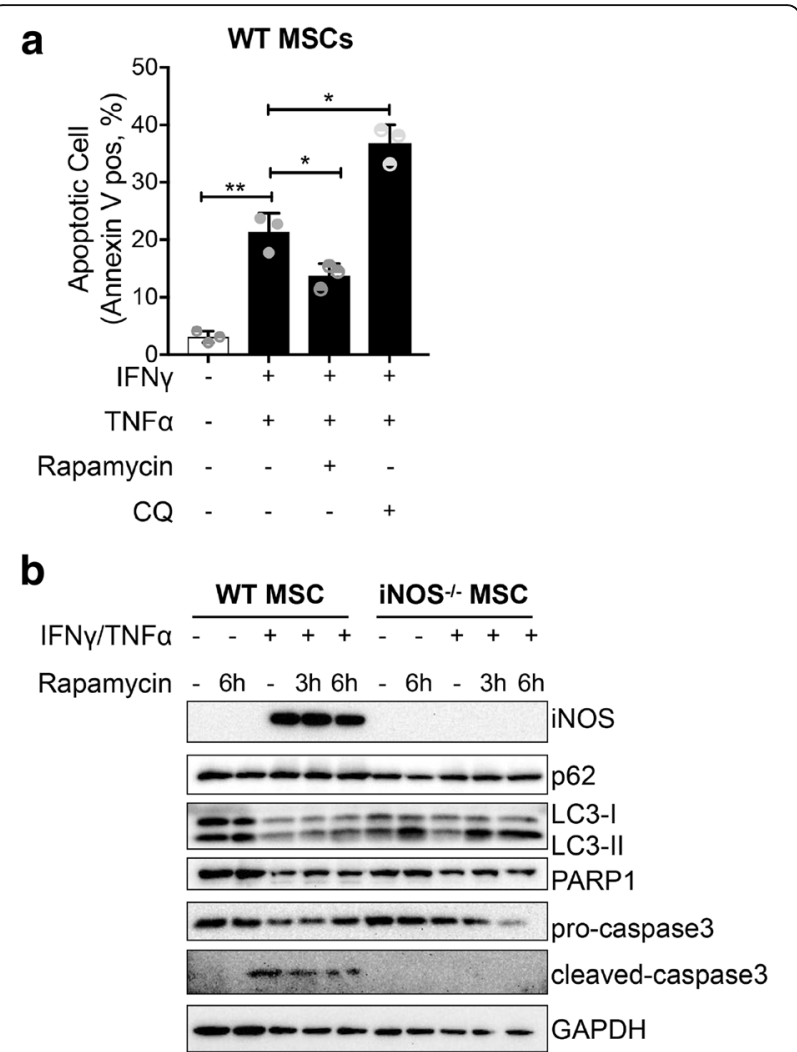

Fig. 5 Blockade of autophagy by iNOS sensitizes BM-MSCs to apoptosis. a Apoptotic cells were analyzed using flow cytometry in MSCs treated or not (ctrl) for $48 \mathrm{~h}$ with IFNY/TNFa $(10 \mathrm{ng} / \mathrm{ml}$ each), alone or in combination with chloroquine $(\mathrm{CQ})$, rapamycin. The percentage of Annexin $\mathrm{V}$-positive cells relative to untreated controls is indicated in a bar chart. $\mathbf{b}$ Representative Western blot of LC3I/ LC3II, p62, PARP, and Caspase-3 from wild-type BM-MSCs and NNOS $^{-1}$ BM-MSCs pretreated or not with IFNY/TNFa (10 ng/ml each) for 16 $\mathrm{h}$ and treated with rapamycin for the indicated time

expression, indicating that increasing autophagic activity reduced ER stress (Additional file 3: Figure S3).

\section{Discussion}

MSCs exhibit potent immunosuppressive and anti-inflammatory activities and have been demonstrated to have therapeutic effects in various diseases and in animal models of GvHD and autoimmune diseases including SLE, experimental autoimmune encephalomyelitis (EAE), and collagen-induced arthritis (CIA) [1, 3]. Additionally, MSCs are also being used in tissue and organ transplantation in several clinical trials, including islet, liver, and renal transplantation [4, 24, 25]. However, the results have often been inconsistent. Many issues remain unresolved. Among them is the varied response to MSC infusions in different patients affected by the same disease. It appears that MSCs can be therapeutically efficacious without being engrafted in the hosts. In fact, the vast majority of infused MSCs reside only transiently in the lungs before becoming undetectable within a few hours [6]. How those MSCs disappear in vivo remains largely elusive. When exposed to IFN $\gamma / \mathrm{TNF} \alpha$, mouse MSCs could undergo apoptosis mediated by Fas and were thus compromised in their bone-repairing function [7]. IFN $\gamma / \mathrm{TNF} \alpha$ were also shown to induce autophagy in MSCs and inhibition of autophagy could augment the immunosuppressive effect on T cells by MSCs [8, 9]. However, survival of MSCs was recently shown to be dispensable for the immunosuppressive effect [10]. These studies suggest that a better understanding of the fate of MSC infusions and the mechanisms underlying MSC therapeutic activity would be highly desirable.

We here demonstrated that the apoptosis of mouse BM-MSCs triggered by the synergistic action of IFN $\gamma$ and TNF $\alpha$ is mediated by NO. Importantly, we observed that IFN $\gamma / \mathrm{TNF} \alpha$ also induced apoptosis in $\mathrm{Fas}^{-/-}$MSCs in an NO-dependent manner, and Fas contributed to IFN $\gamma /$ TNF $\alpha$-induced cell death only when a Fas agonist was added (schematic representation described in Fig. 6). Moreover, IFN $\gamma / \mathrm{TNF} \alpha$-induced expression of Fas also depended on NO, and consequently, Fas agonist-induced cell death was greatly attenuated in $\mathrm{iNOS}^{-/-}$MSCs. Our finding is thus in contrast to the previous report showing Fas pathway as a mediator of the cell death caused by the two cytokines [7]. Future studies are needed to resolve this discrepancy.

$\mathrm{NO}$ is a potent and pleiotropic free radical molecule that has been involved in a wide variety of physiological and pathophysiological functions [26]. NO and NO-derived reactive nitrogen species can interact with many receptors, ion channels, and enzymes [27]. NO is generated in low levels by two constitutive NOS (eNOS and nNOS) and in much greater levels by the iNOS [28]. $\mathrm{NO}$ is a ubiquitous cellular messenger molecule in the cardiovascular, nervous, and immune systems, where $\mathrm{NO}$ is capable of eliciting a multitude of physiological responses, such as blood flow regulation and tissue responses to hypoxia [29]. At high concentrations, NO inhibits TCR-induced $\mathrm{T}$ cell proliferation and cytokine production [30]. However, there has been a long debate about the specific role that NO plays in apoptosis. It has been shown that NO could participate in the apoptosis process by either inhibiting or promoting some apoptotic events. Several studies have referred that NO is a novel and potent inhibitor of apoptosis. Endogenous NO synthesis or exposure to low levels of NO donors has been demonstrated to inhibit apoptosis in human B lymphocytes, splenocytes, eosinophils, and endothelial cells [31-34]. NOS inhibitors have also been directed toward the specific disruption of the Fas-induced apoptotic mechanism. Basal NOS activity in human leukocytes has been revealed to inhibit Fas-induced apoptosis via a 


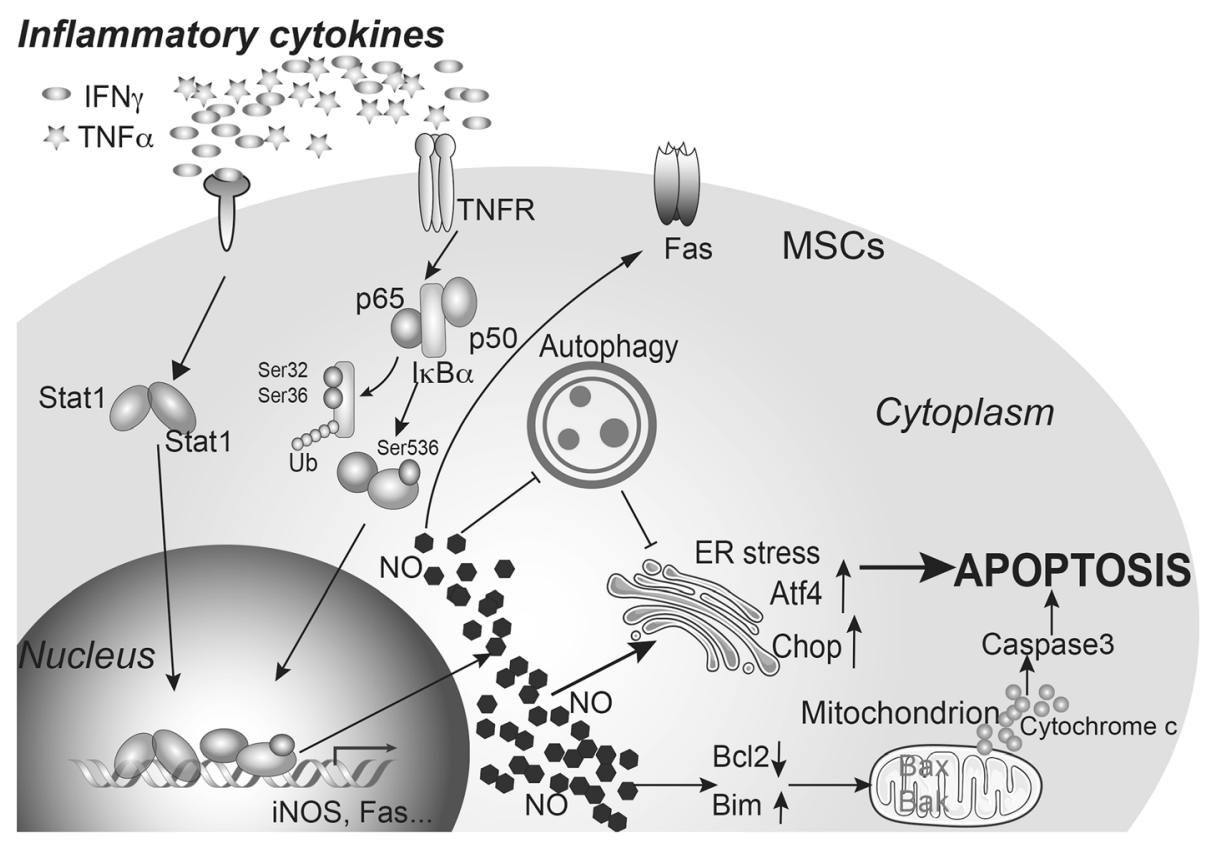

Fig. 6 The role of iNOS in IFNY/TNFa-induced apoptosis of MSCS. MSCS undergo apoptosis due to the synergistic action of IFNY and TNFa under an inflammatory microenvironment. The pro-inflammatory cytokines IFNY and TNFa induce apoptosis of mouse BM-MSCs in an NO-dependent manner. NO impairs autophagy in BM-MSCs and contributes to ER stress. NO also upregulates Fas and enhances Fas-mediated apoptosis

cGMP-independent mechanism and further inhibition of caspase activation $[35,36]$. Conversely, an excess of NO is believed to play a causal role in demise of several type of cells, including neuronal cells [13], T cells [14], dendritic cells [15], and microglial cells [16]. The involvement of Fas/FasL system and signal transduction pathway in NO-induced apoptosis in human lymphoid cells has been examined, suggesting that NO triggers the death receptor system by regulating the expression of ligands involved in apoptosis [37]. Herein, our results with mouse MSCs clearly demonstrate that NO, far from protecting MSCs from Fas-induced apoptosis, synergized with the Fas agonist antibody Jo2 in the induction of apoptosis. The dichotomy in the function of NO may reflect divergence among different cell types and needs to be considered when making strategies toward the use of NO donors as therapeutic agents.

Autophagy, the basic catabolic process, occurs at basal levels in most tissues and contributes to routine cell recycling by lysosomes. During this process, substances in the cytoplasm are phagocytosed by autophagosomes, which are transported to the lysosomes for degradation. The degradation products can be reused in the syntheses of macromolecules and in energetic metabolism. Autophagy plays an important role in cell survival process [38]. Early studies suggested that autophagy serves as a cell survival mechanism in some pathological processes via its suppressive role in necroptosis and PARP-mediated apoptosis during unfavorable growth conditions or cellular stress [39]. Autophagy plays an important role in suppressing apoptosis in retinal ganglion cells. It has also been observed that activation of autophagy could promote retinal ganglion cell survival and that inhibition of autophagy can reduce cell survival during optic nerve degeneration [40]. ER plays a major role in the synthesis, folding, and structural maturation of more than a third of all proteins made in the cell [41]. ER stress is caused by the accumulation of misfolded proteins in the $E R$, which triggers an adaptive program. Autophagy may alleviate the ER stress triggered by damaged organelles [42]. In this study, we also showed that the generation of NO in MSCs stimulated by IFN $\gamma / \mathrm{TNF} \alpha$ impairs autophagy, which aggravates ER stress and promotes apoptosis. The finding that NO can impair pro-survival autophagy also renders support to this notion [11]. Collectively, our data indicate that inhibition of autophagy by NO induced by inflammatory cytokines plays a pro-apoptotic role in MSCs and it might not benefit the survival of MSCs when they are transplanted for inflammatory diseases.

Our study only focuses on the effects of NO induced by inflammatory cytokines on the fate of MSCs in vitro, whether NO-induced apoptosis of mouse MSCs accounts for the disappearance of MSCs in vivo after infusions in inflammatory diseases remains unclear. However, while deletion or inhibition of iNOS can potentially prolong the survival of mouse MSCs in vivo, the immunosuppressive function of MSCs thus obtained may also be compromised because $\mathrm{NO}$ is essential for the inhibition of the T cells. 


\section{Conclusions}

Our studies show that the pro-inflammatory cytokines IFN $\gamma$ and TNF $\alpha$ synergistically induce apoptosis of mouse BM-MSCs in an NO-dependent manner. NO was found to impair autophagy in BM-MSCs and to contribute to ER stress. NO also upregulates Fas and enhances Fas-mediated apoptosis. These findings provide an important insight into the mechanism by which mouse MSCs undergo apoptosis in the presence of pro-inflammatory cytokines and should bear implications in MSC-based therapy.

\section{Additional files}

Additional file 1: Figure S1. Effect of a combination of IFNY and TNFa on the inhibition of autophagy in MSCs. Representative Western blot of LC3I/LC3II and p62 from both wild-type and iNOS ${ }^{-1-}$ BM-MSCs pretreated or not with IFNy/TNFa (10 ng/ml each) for $16 \mathrm{~h}$ and treated with chloroquine (CQ) for the indicated time. (TIF $212 \mathrm{~kb}$ )

Additional file 2: Figure S2. IFNy/TNFa induce ER stress in BM-MSCs dependently iNOS activity. ( $\mathrm{a}$ and b) Both wide type and $\mathrm{Fas}^{-1-}, \mathrm{BM}-\mathrm{MSCS}$ were treated with IFNY/TNFa (10 ng/ml each) in the absence or presence (1 h pre-incubation) of L-NMMA (1 mM) for $24 \mathrm{~h}$, ER stress-related transcripts were quantified by real-time PCR. (TIF $316 \mathrm{~kb}$ )

Additional file 3: Figure S3. ER stress was upregulated by blocking autophagy. MSCs were treated or not (mock) for $48 \mathrm{~h}$ with IFNY/TNFa (10 ng/ml each), alone or in combination with chloroquine, rapamycin. ER stress-related gene transcripts were quantified by real-time PCR. (TIF 645 kb)

\section{Abbreviations}

AlF: Apoptosis-inducing factor; CIA: Collagen-induced arthritis; CQ: Chloroquine; EAE: Experimental autoimmune encephalomyelitis; ER: Endoplasmic reticulum; GvHD: Graft-versus-host disease; iNOS: Inducible nitric oxide synthase; L-NMMA: $N^{G}$-monomethyl-L-arginine;

MSCs: Mesenchymal stem/stromal cells; NO: Nitric oxide; SLE: Systemic lupus erythematosus; SNAP: S-Nitroso-N-acetyl-penicillamine; UPR: Unfolded protein response; YY1: Yin Yang 1

\section{Acknowledgements}

Not applicable.

\section{Funding}

This work was supported in part by grants from the National Natural Science Foundation of China $(81530043,81672797,31771260$, and 81702869), the National Key R\&D Program of China (2018YFA0107500), the China Postdoctoral Science Foundation (2016M591917), and the Postdoctoral Science Foundation of Jiangsu Province (1501063A).

\section{Availability of data and materials}

Please contact the corresponding authors for materials.

\section{Authors' contributions}

$X L, Y S$, and CS contributed to the study conception and design and financial support. $\mathrm{XL}, \mathrm{BS}$, and $\mathrm{YL}$ performed the experiments and contributed to the collection and assembly of the data, and data analysis and interpretation. $\mathrm{XL}$ created the graphs. $\mathrm{XL}$ and CS contributed to the data interpretation, manuscript writing, and final approval of the manuscript. All authors analyzed and interpreted the data. All authors read and approved the final manuscript.

\section{Ethics approval and consent to participate}

Not applicable.

\section{Consent for publication}

Not applicable.

\section{Competing interests}

The authors declare that they have no competing interests.

\section{Publisher's Note}

Springer Nature remains neutral with regard to jurisdictional claims in published maps and institutional affiliations.

Received: 1 November 2018 Revised: 4 December 2018 Accepted: 6 December 2018 Published online: 11 January 2019

\section{References}

1. Wang $Y$, et al. Plasticity of mesenchymal stem cells in immunomodulation: pathological and therapeutic implications. Nat Immunol. 2014;15(11):1009-16.

2. Shi $Y$, et al. Tumour-associated mesenchymal stem/stromal cells: emerging therapeutic targets. Nat Rev Drug Discov. 2017;16(1):35-52.

3. Shi $Y$, et al. How mesenchymal stem cells interact with tissue immune responses. Trends Immunol. 2012;33(3):136-43.

4. Shi $Y$, et al. Immunoregulatory mechanisms of mesenchymal stem and stromal cells in inflammatory diseases. Nat Rev Nephrol. 2018;14(8):493-507.

5. Ren $\mathrm{G}$, et al. Mesenchymal stem cell-mediated immunosuppression occurs via concerted action of chemokines and nitric oxide. Cell Stem Cell. 2008; 2(2):141-50.

6. Lee $\mathrm{RH}$, et al. Intravenous hMSCs improve myocardial infarction in mice because cells embolized in lung are activated to secrete the antiinflammatory protein TSG-6. Cell Stem Cell. 2009;5(1):54-63.

7. Liu Y, et al. Mesenchymal stem cell-based tissue regeneration is governed by recipient T lymphocytes via IFN-gamma and TNF-alpha. Nat Med. 2011; 17(12):1594-601.

8. Dang $\mathrm{S}$, et al. Autophagy promotes apoptosis of mesenchymal stem cells under inflammatory microenvironment. Stem Cell Res Ther. 2015;6:247.

9. Dang $\mathrm{S}$, et al. Autophagy regulates the therapeutic potential of mesenchymal stem cells in experimental autoimmune encephalomyelitis. Autophagy. 2014;10(7):1301-15.

10. Galleu A, et al. Apoptosis in mesenchymal stromal cells induces in vivo recipient-mediated immunomodulation. Sci Transl Med. 2017;9(416): eaam7828.

11. Sarkar $\mathrm{S}$, et al. Complex inhibitory effects of nitric oxide on autophagy. Mol Cell. 2011;43(1):19-32.

12. Kim H, et al. Hierarchical regulation of mitochondrion-dependent apoptosis by BCL-2 subfamilies. Nat Cell Biol. 2006;8(12):1348-58.

13. Gu Z, et al. Redox reactions induced by nitrosative stress mediate protein misfolding and mitochondrial dysfunction in neurodegenerative diseases. Mol Neurobiol. 2010:41(2-3):55-72

14. Saio M, et al. Tumor-infiltrating macrophages induce apoptosis in activated CD8(+) T cells by a mechanism requiring cell contact and mediated by both the cell-associated form of TNF and nitric oxide. J Immunol. 2001; 167(10):5583-93.

15. Lu L, et al. Induction of nitric oxide synthase in mouse dendritic cells by IFN-gamma, endotoxin, and interaction with allogeneic T cells: nitric oxide production is associated with dendritic cell apoptosis. J Immunol. 1996; 157(8):3577-86.

16. Mayo L, Stein R. Characterization of LPS and interferon-gamma triggered activation-induced cell death in N9 and primary microglial cells: induction of the mitochondrial gateway by nitric oxide. Cell Death Differ. 2007;14(1):183-6.

17. Itoh $\mathrm{N}$, et al. The polypeptide encoded by the cDNA for human cell surface antigen Fas can mediate apoptosis. Cell. 1991;66(2):233-43.

18. Yonehara $\mathrm{S}$, et al. A cell-killing monoclonal antibody (anti-Fas) to a cell surface antigen co-downregulated with the receptor of tumor necrosis factor. J Exp Med. 1989;169(5):1747-56.

19. Hess DT, et al. Protein S-nitrosylation: purview and parameters. Nat Rev Mol Cell Biol. 2005;6(2):150-66.

20. Garban HJ, Bonavida B. Nitric oxide inhibits the transcription repressor yinYang 1 binding activity at the silencer region of the Fas promoter: a pivotal role for nitric oxide in the up-regulation of Fas gene expression in human tumor cells. J Immunol. 2001;167(1):75-81.

21. Hongo $F$, et al. Inhibition of the transcription factor yin Yang 1 activity by S-nitrosation. Biochem Biophys Res Commun. 2005;336(2):692-701.

22. Cybulsky AV. Endoplasmic reticulum stress, the unfolded protein response and autophagy in kidney diseases. Nat Rev Nephrol. 2017;13(11):681-96.

23. Klionsky DJ, et al. Guidelines for the use and interpretation of assays for monitoring autophagy (3rd edition). Autophagy. 2016;12(1):1-222. 
24. Chen C, Hou J. Mesenchymal stem cell-based therapy in kidney transplantation. Stem Cell Res Ther. 2016;7:16.

25. Casiraghi $F$, et al. Mesenchymal stromal cells in renal transplantation: opportunities and challenges. Nat Rev Nephrol. 2016;12(4):241-53.

26. Stamler JS, et al. Biochemistry of nitric oxide and its redox-activated forms. Science. 1992;258(5090):1898-902.

27. Edwards TM, Rickard NS. New perspectives on the mechanisms through which nitric oxide may affect learning and memory processes. Neurosci Biobehav Rev. 2007;31(3):413-25.

28. Billiar TR. Nitric oxide. Novel biology with clinical relevance. Ann Surg. 1995; 221(4):339-49.

29. Foster MW, et al. Protein S-nitrosylation in health and disease: a current perspective. Trends Mol Med. 2009;15(9):391-404.

30. Hoffman RA, et al. Differential modulation of CD4 and CD8 T-cell proliferation by induction of nitric oxide synthesis in antigen presenting cells. Transplantation. 2002;74(6):836-45.

31. Mannick JB, et al. Nitric oxide produced by human B lymphocytes inhibits apoptosis and Epstein-Barr virus reactivation. Cell. 1994;79(7):1137-46.

32. Hebestreit $\mathrm{H}$, et al. Disruption of fas receptor signaling by nitric oxide in eosinophils. J Exp Med. 1998;187(3):415-25.

33. Genaro AM, et al. Splenic B lymphocyte programmed cell death is prevented by nitric oxide release through mechanisms involving sustained Bcl-2 levels. J Clin Invest. 1995;95(4):1884-90.

34. Dimmeler S, et al. Suppression of apoptosis by nitric oxide via inhibition of interleukin-1 beta-converting enzyme (ICE)-like and cysteine protease protein (CPP)-32-like proteases. J Exp Med. 1997;185(4):601-7.

35. Kim YM, et al. Nitric oxide inhibits apoptosis by preventing increases in caspase-3-like activity via two distinct mechanisms. J Biol Chem. 1997; 272(49):31138-48.

36. Mannick JB, et al. Nitric oxide inhibits Fas-induced apoptosis. J Biol Chem. 1997;272(39):24125-8.

37. Chlichlia $\mathrm{K}$, et al. Caspase activation is required for nitric oxide-mediated, CD95(APO-1/Fas)-dependent and independent apoptosis in human neoplastic lymphoid cells. Blood. 1998;91(11):4311-20.

38. Kroemer G, Levine B. Autophagic cell death: the story of a misnomer. Nat Rev Mol Cell Biol. 2008;9(12):1004-10.

39. Boya P, et al. Inhibition of macroautophagy triggers apoptosis. Mol Cell Biol. 2005;25(3):1025-40.

40. Rodriguez-Muela $\mathrm{N}$, et al. Autophagy promotes survival of retinal ganglion cells after optic nerve axotomy in mice. Cell Death Differ. 2012;19(1):162-9.

41. Anelli T, Sitia R. Protein quality control in the early secretory pathway. EMBO J. 2008;27(2):315-27.

42. Cebollero $\mathrm{E}$, et al. Reticulophagy and ribophagy: regulated degradation of protein production factories. Int J Cell Biol. 2012;2012:182834.

Ready to submit your research? Choose BMC and benefit from:

- fast, convenient online submission

- thorough peer review by experienced researchers in your field

- rapid publication on acceptance

- support for research data, including large and complex data types

- gold Open Access which fosters wider collaboration and increased citations

- maximum visibility for your research: over $100 \mathrm{M}$ website views per year

At $\mathrm{BMC}$, research is always in progress.

Learn more biomedcentral.com/submissions 